\title{
Burning Characteristics of a Combustible Liquid Soaked in Porous Beds
}

\author{
TOMOMITSU TAKEUCHI, TAKASHI TSURUDA, SATORU ISHIZUKA \\ and TOSHISUKE HIRANO
}

Department of Reaction Chemistry

The University of Tokyo

7-3-1 Hongo, Bunkyo-ku, Tokyo, Japan

ABSTRACT

An experimental study has been made on the burning characteristics of liquid methanol soaked in porous beds of glass beads. In order to study the effects of the soil properties on the burning characteristics, experiments were carried out for several bead diameters. The consumption rates of methanol and the temperature profiles in the porous beds were measured.

The results showed that the total weight of the methanol consumed was only about half of the initial weight and the consumption rate decreased with time. The total weight of the consumed methanol and the consumption rate of methanol increased with decreasing bead diameter.

The temperature profiles indicated that the dry region in a porous bed, in which only methanol vapor was assumed to exist, expanded downward with time. The discrepancy between the lower boundary of the dry region and the liquid level evaluated on the basis of methanol consumption for small beads suggested that the liquid moved upward by a capillary force.

A simplified heat transfer model was proposed for large beads and the predicted results agreed qualitatively with the experimental ones. The consumption rate on large beads bed was inferred to be controlled mainly by the heat transfer in the porous bed.

KEY WORDS: liquid combustion, burning characteristics, fuel soaked porous bed.

\section{INTRODUCTION}

When a storage tank of liquid fuel is accidentally damaged or corroded, the liquid fuel leaks from the tank and soaks into soil. If it is ignited, a fire will occur. The scale and intensity of the fire depend not only on the amount of soaked liquid, but also on the properties of the soil. In order to predict the damage in such a hazard, it is important to study the effects of the soil properties on the burning characteristics of the liquid. Although the flame spread mechanisms over porous solids soaked with combustible liquid have been extensively studied [1-4], the burning characteristics such as the burning time or the burning rate have not yet been studied in detail. Thus, we study the burning characteristics of a 
combustible liquid soaked in porous materials using a bed of glass beads.

\section{EXPERIMENTAL METHODS AND MATERIALS}

A schematic diagram of the experimental apparatus is shown in Fig. 1. A stainless-steel cylindrical vessel with a diameter of $0.1 \mathrm{~m}$ and a height of $0.1 \mathrm{~m}$ was used. As in the previous studies [1,3], glass beads of various diameters were packed in the vessel and used as a porous bed. The diameters of the bead (d) were $0.2,0.5,1.4,3$, and $5 \mathrm{~mm}$. Methanol (99\% pure) was used as a combustible liquid. The porosities of the beds are about $35-36 \%$ for $d=0.2,0.5,1.4$ and $3 \mathrm{~mm}$ and $38.5 \%$ for $d=5 \mathrm{~mm}$. The vessel was counter-balanced by a weight and the variation of the methanol weight in the vessel was measured by using a strain-gauge.

The temperature distributions in the porous beds were measured by $\mathrm{C}-\mathrm{A}$ thermocouples (wire diameter $0.1 \mathrm{~mm}$ ) installed in the vessel. In order to obtain the axial temperature profiles, four thermocouples were installed on the center axis of the vessel at the points of $z=0,-6.5,-11.5$, and $-20 \mathrm{~mm}(z$ is the distance upward from the surface). In order to obtain the radial temperature profiles, four thermocouples were installed at the positions of $r=0,17,33$ and $46 \mathrm{~mm}$ ( $r$ is the radial distance from the center) at a plane $6.5 \mathrm{~mm}$ beneath the surface.

Experiments were made under a natural convection condition and at room temperature $(296-300 \mathrm{~K})$.

\section{RESULTS}

\section{Appearance of Flame}

When ignited, a flame immediately spreads over the bed and a blue diffusion flame is established. The flame is anchored at the rim of the vessel and the flame shape is almost conical. Initially, the flame height is over $20 \mathrm{~cm}$ and the flame is flickering with a frequency of $56 \mathrm{~Hz}$. As the combustion proceeds, the flame height gradually decreases, j.e., the flame approaches the surface of the bed, resulting in a flat-shaped flame on the surface. Later, the flame is locally extinguished and some parts of the bed become uncovered with the flame. Finally, the flame completely extinguishes. The times from the ignition to the complete extinction are

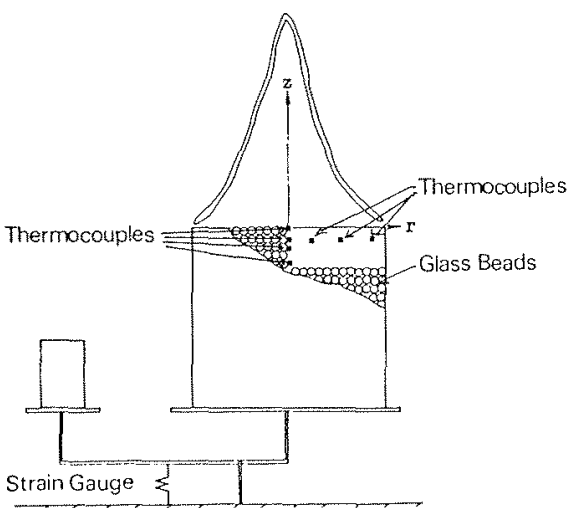

FIGURE 1. Schematic diagram of experimental apparatus

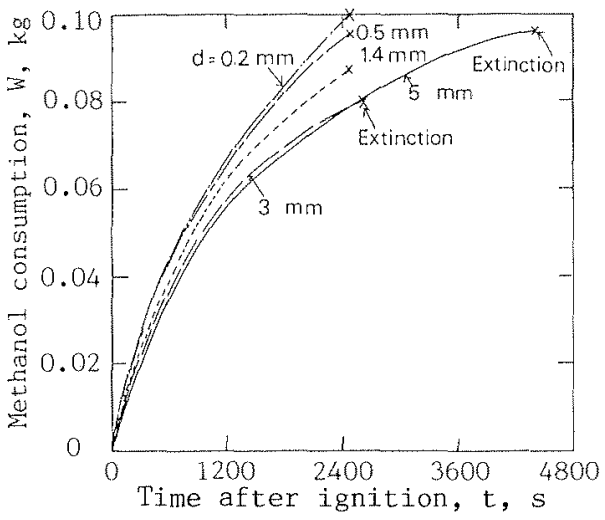

FIGURE 2. Methanol consumption at various bead diameters 
almost the same (about $2500 \mathrm{~s}$ ) for $\mathrm{d}=0.2,0.5,1.4$ and $3 \mathrm{~mm}$, but the time is longer (about $4500 \mathrm{~s}$ ) for $\mathrm{d}=5 \mathrm{~mm}$.

Consumption Rate of Methanol

Figure 2 shows the methanol consumption for various bead diameters. With increasing the bead diameter, the rate of the decrease of the methanol weight gradually decreases.

Near extinction, the methanol weights consumed are $0.1 \mathrm{~kg}$ for $\mathrm{d}=0.2$ $\mathrm{mm}, 0.08 \mathrm{~kg}$ for $d=3 \mathrm{~mm}$ (at $t \simeq 2500 \mathrm{~s}$ ), and $d=0.092 \mathrm{~kg}$ for $d=5 \mathrm{~mm}$ (at $t \simeq 4500$ s). Several runs were made for each bead diameter. The scatters in the methanol weights consumed were very small (within 3\%) for each bead diameter. Since the initial weight of the methanol in the vessel was about $0.2 \mathrm{~kg}$, these values indicate that only about a half of the initial methanol was burned on the bed.

Based on the results in Fig.2, the consumption rates of methano1, $R$ were derived and shown in Fig.3. It is seen in Fig.3 that the consumption rates decrease with time. The consumption rates at ignition are about $8 \times 10^{-5} \mathrm{~kg} / \mathrm{s}$ and those at extinction are about $1.5 \times 10^{-5} \mathrm{~kg} / \mathrm{s}$. If we evaluate the linear regression rates of the methanol in the vessel by dividing the consumption rate ( $R$ ) by the density of methanol and by the cross sectional area of the pore of the vessel, the values are $3 \times 10^{-5} \mathrm{~m} / \mathrm{s}$ and $0.6 \times 10^{-5} \mathrm{~m} / \mathrm{s}$ at ignition and extinction, respectively. These linear regression rates are about the same order of the linear regression rate at liquid methanol burning in a $0.1 \mathrm{~m}$ diameter pool $\left(1.7 \times 10^{-5} \mathrm{~m} / \mathrm{s}\right)[5-9]$. It is interesting to note that except for $d=5 \mathrm{~mm}$, the value of $\mathrm{R}$ becomes larger as the bead diameter is decreased in the range of conditions at the present experiments.

Figure 4 shows the relation between the flame height, $h$ and the consumption rate, $R$. The values at $t=120,300,600$, and $900 \mathrm{~s}$ are indicated in Fig.4. It is seen in Fig. 4 that the flame height almost linearly decreases with the decreasing consumption rate.

Temperature Distribution in the Porous Bed

Figure 5 shows the variation of temperature in the $5 \mathrm{~mm}$ beads bed
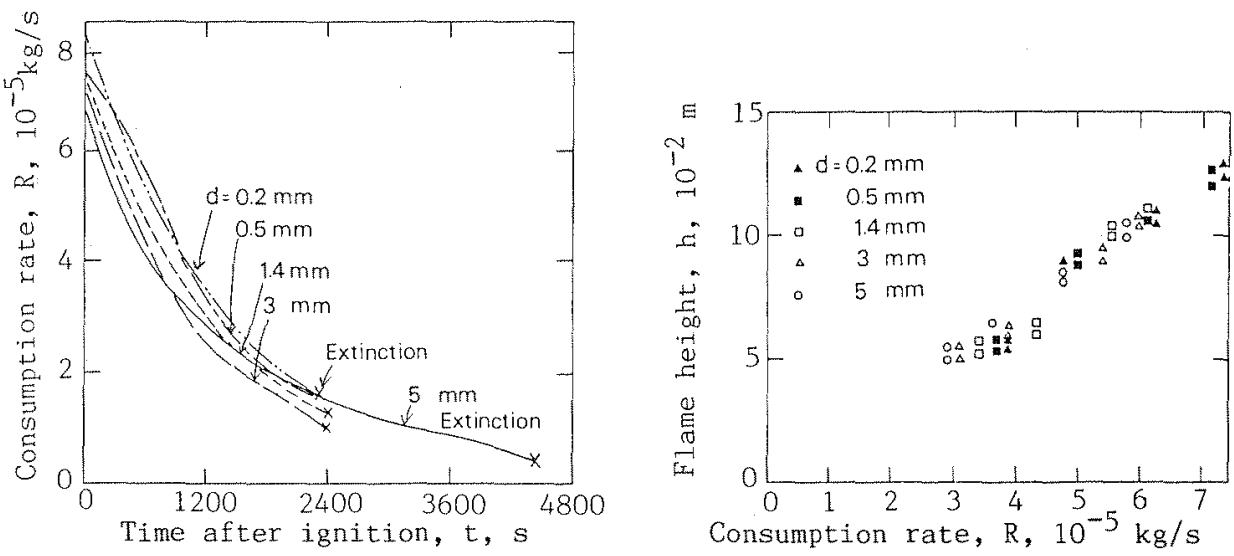

FIGURE 3. Consumption rate of methanol

FIGURE 4. Relation between flame height and consumption rate 
measured by the four thermocouples installed on the center axis of the vessel $(r=0 \mathrm{~mm})$. The temperature on the surface $(z=0 \mathrm{~mm})$ immediately rises after ignition, attains its maximum of $740 \mathrm{~K}$ at $t=2100 \mathrm{~s}$ and then decreases. At $6.5 \mathrm{~mm}$ beneath the surface, the temperature first increases rapidly, but the rate of increase slows down around $335 \mathrm{~K}$, and then the temperature increases rapidly again. At a much deeper position $(z=-11.5$ $\mathrm{mm})$, the temperature increases first, but again the rate of increase slows down around $335 \mathrm{k}$, and then steeply increases. The retardation of the temperature increase around $335 \mathrm{~K}$ is seen also in the temperature profile at $z=-20 \mathrm{~mm}$. The fact that the temperature $(335 \mathrm{~K})$ at which the retardatiop occurs is very close to the boiling point of methanol ( $337.9 \mathrm{~K}$ at $1.01 \times 10^{5} \mathrm{~Pa}$ ) implies that the abrupt temperature increase is due to the completion of the methanol evaporation in the region of the thermocouple. This suggests that the region in which the temperature is higher than about $335 \mathrm{~K}$ is a dry region [3] in which only methanol vapor exists in the pore.

From the temperature-time records, the axial and radial temperature profiles in the bed are obtained. Figure 6 shows the result, with elapsed time as a parameter. As can be seen in the radial temperature profile (Fig. $6(\mathrm{a}))$ at $t=270 \mathrm{~s}$, when the flame is anchored at the rim, the temperature near the center at $z=-6.5 \mathrm{~mm}$ is almost uniform and increases near the wall. At $t=600 \mathrm{~s}$, an overall temperature profile is similar to that at $t=270 \mathrm{~s}$ except for the absolute value of the temperature which increases with time. As the combustion process proceeds, the flame height decreases and the rate of the temperature increase in the near-wall region becomes smaller than that in the central region. The temperature increase in the near-wall region stops at $t \simeq 1000 \mathrm{~s}$, when the flame base starts to leave the rim. During $t=1000 \sim 2000 \mathrm{~s}$, the wall temperature stays constant, while the temperature except for the near-wall region continues to increase.

In the axial temperature profile (Fig.6(b)), we can find that the temperature at $r=0 \mathrm{~mm}$ increases with the distance to the surface decreases. At $t=270 \mathrm{~s}$, the temperature at $z=-6.5 \mathrm{~mm}$ is very close to the boiling point of methanol and the temperature gradient between $z=0 \mathrm{~mm}$ and $a=-6.5 \mathrm{~mm}$ is much larger than that between $z=-6.5 \mathrm{~mm}$ and $z=-20 \mathrm{~mm}$. This suggests that the bed is roughly divided into two regions by a surface, above which only methanol vapor exists. The position where the temperature is the boiling point of methanol is considered to correspond to the surface of the liquid. The surface is seen to be located around $z=-6.5 \mathrm{~mm}$ at $t=270 \mathrm{~s}$ and around $z=-11.5 \mathrm{~mm}$ at $\mathrm{t}=600 \mathrm{~s}$.

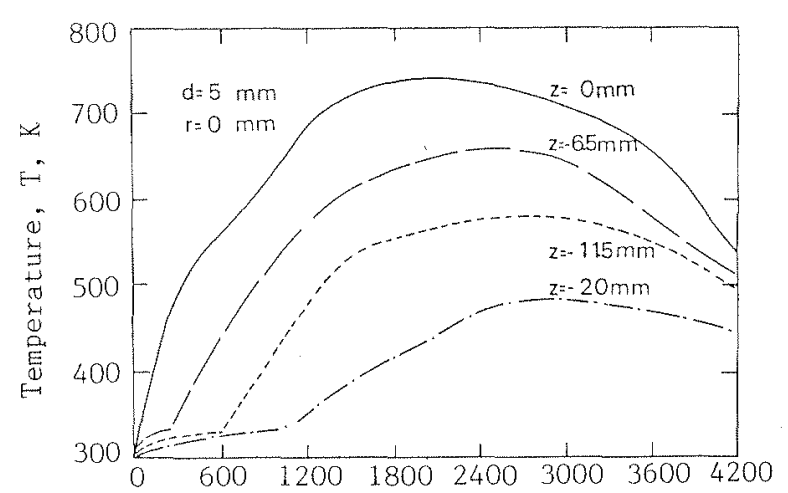

At $t=1200 \mathrm{~s}$, the four points fall on a straight 1ine. Further, the radial temperature profile (at $z=-6.5 \mathrm{~mm}$ ) in Fig.6(a) is almost uniform at $t=1200$ s. These facts imply that the heat transfer in the region is steady and onedimensiona1. Based on the similar phenomena at $t=1800 \mathrm{~s}$, also the heat transfer can be assumed to be steady and onedimensional.

Time after ignition, $t, s$

FIGURE 5. Variation of the temperature in the porous bed 


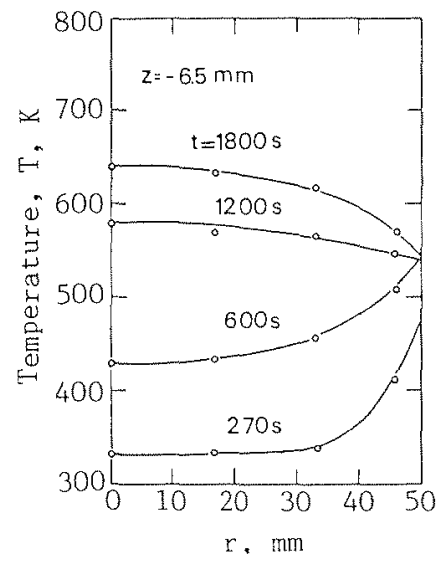

(a) Radial temperature profile

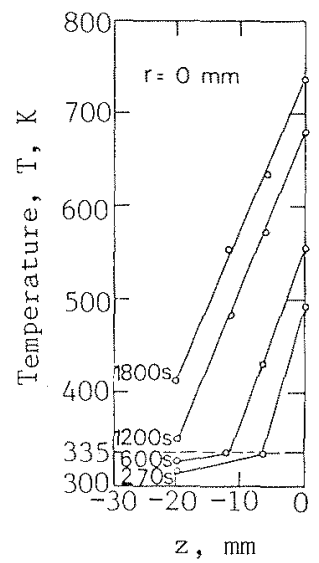

(b) Axial temperature profile

FIGURE 6. Temperatrue distributions in the porous bed

\section{Lower Boundary of the Dry Region}

From the temperature distributions, it is found that the porous bed can be divided into two layers by the surface above which only methanol vapor exists. Therefore this surface can be considered to be the lower boundary of the dry region. Since the surface seems to be almost horizontal, we consider that the position of $335 \mathrm{~K}$ on the center axis is that of the surface. The positions of the surface at the various bead diameters are shown in Fig.7. For comparison, the liquid levels in the porous solids are evaluated on the basis of results on the methanol consumption (Fig.2) and shown by broken lines in this figure. In this evaluation, we assume that the bed is divided into two regions, the upper dry region where only vapor exists and the lower region where only liquid

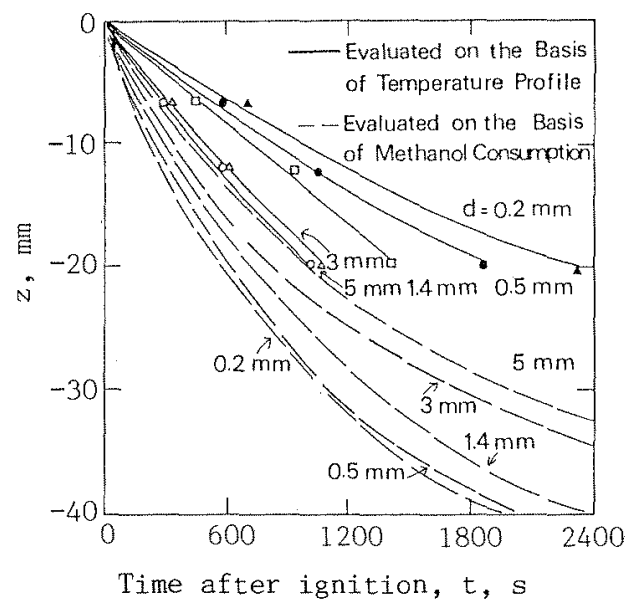
methanol exists in the pore of the beads, and the boundary between the two regions can be simply obtained by dividing the weight of methano 1 consumed by the crosssectional area of the pore of the vessel.

It is seen in Fig.7 that when the bead diameter is large $(\mathrm{d}=3$ $\mathrm{mm}$ and $5 \mathrm{~mm}$ ) the liquid level simply evaluated from the methano 1 consumption agrees well with the boundary obtained from the temperature distribution.

FIGURE 7. Positions of the dry region boundary at various bead diameters 
When the bead diameter is $\operatorname{small}(\mathrm{d}=0.2 \mathrm{~mm}, 0.5 \mathrm{~mm}$, and $1.4 \mathrm{~mm})$, however, they do not coincide with each other. This suggests that when the bead diameter is large, a liquid layer and a vapor layer are clearly divided and when the bead diameter is small, however, the division is not so clear; the liquid can move upward owing to a capillary force and there exists a region where liquid methanol and methanol vapor coexist in the bed. As the bead diameter is decreased, the capillary force becomes larger, thus the location of the lower boundary of the dry region is considerably raised up. The difference in the liquid behavior leads to the difference of the burning characteristics with bead diameters observed in Fig. 2 .

\section{Simplified Model}

When the bead diameter is large, the porous bed can be divided into two regions; the upper region filled with vapor and the lower region filled with liquid. It can be assumed that the boundary between the two regions is almost flat and that the location moves with time. Although the total weight of consumed methanol or the consumption rate varies about $25 \%$ in the range of the conditions at the present experiments, we propose a simplified model to predict the consumption rate of methanol in the bed.

The model adopted here is schematically shown in Fig. 8 . In this model, we consider only heat conduction in the porous bed and neglect the motion of liquid induced by a capillary force in the bed. For simplicity, the following assumptions are made.

(1) Only heat conduction in the axial ( $z$ ) direction is considered and heat conduction in the radial ( $r$ ) direction is neglected. This assumption may be valid especially when several minutes are passed after ignition (see Fig.6(a) and Fig.6(b)).

(2) The beads bed in the vessel is divided into two regions; the upper region (dry region) where only methanol vapor exists and the lower region only liquid methanol exists.

(3) The temperature at the boundary surface between the upper region and the lower region equals to the boiling point of methanol $\theta_{b}$.

(4) The temperatures at the top and the bottom of the bed are constant and given as $\theta_{f}$ and $\theta_{0}$, respectively.

(5) Based on the fact that the temperature increase at the bottom is very small (less than $1 \mathrm{~K}$ ), the bottom of the bed is assumed to be situated in the infinity.

Based on the above assumptions, the governing equations for the upper region and the lower region are given by

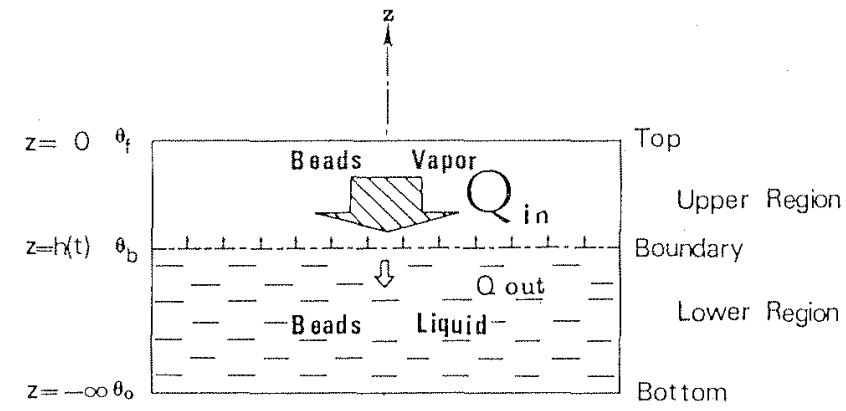

FIGURE 8. Schematic diagram of the model 
$\frac{\partial \theta_{1}}{\partial t}=a_{1} \frac{\partial^{2} \theta_{1}}{\partial z^{2}}$,
$\frac{\partial \theta_{2}}{\partial t}=a_{2} \frac{\partial^{2} \theta_{2}}{\partial z^{2}}$,

and the boundary conditions and the initial condition are

$$
\begin{array}{ll}
\text { at } z=0 ; & \theta_{1}(t, z)=\theta_{f}, \\
\text { at } z=h(t) ; & \theta_{1}(t, z)=\theta_{2}(t, z)=\theta_{b}, \\
\text { as } z \rightarrow-\infty ; & \theta_{2}(t, z)=\theta_{0},
\end{array}
$$

and

for $t=0$;

$$
\theta_{1}(t, z)=\theta_{2}(t, z)=\theta_{0},
$$

where $\theta_{1}(t, z)$ and $\theta_{2}(t, z)$ are the temperatures of the upper region and the lower region, respectively, $a_{1}$ and $a_{2}$ are the thermal diffusivities of the upper region and the lower region, respectively, and $h(t)$ is the location of the boundary surface between the two regions.

The solutions, $\theta_{1}(t, z)$ and $\theta_{2}(t, z)$, can be determined by solving the second order partial differential equations with individual set of two boundary conditions $\left(\theta_{1}(t, 0)\right.$ and $\theta_{1}(t, h)$ for $\theta_{1}, \theta_{2}(t, h)$ and $\theta_{2}(t,-\infty)$ for $\left.\theta_{2}\right)$ and an initial condition. However, note that the location of the boundary $h(t)$ is not specified. To determine the solutions uniquely, we need an additional condition, which is given as follows. During a fraction $\Delta t$ of time $t$, a heat $Q_{\text {in }}$ conducted from the upper region into the boundary surface through a total cross-sectional area $S$ of the vessel is $\lambda_{1}\left(\partial \theta_{1} / \partial z\right) S \Delta t$ and a heat $Q_{\text {out }}$ conducted away from the boundary surface into the lower region $1 S \lambda_{2}\left(\partial \theta_{2} / \partial z\right) S \Delta t$. As the net heat is used to vaporize the methanol at the boundary surface, the following relation can be held at the boundary surface.

$\lambda_{1} \frac{\partial \theta_{1}}{\partial z}-\lambda_{2} \frac{\partial \theta_{2}}{\partial z}=\varepsilon \rho q \frac{\Delta z}{\Delta t}$,

where $\lambda_{1}$ and $\lambda_{2}$ are the thermal conductivities of the upper region and the lower region, respectively, $\varepsilon$ is the porosity of the beads, $p$ is the density of the methanol, and $\mathrm{q}$ is the latent heat of methanol.

If we introduce a nondimensional coordinate $n=z / 2\left(a_{1} t\right)^{1 / 2}$ and assume the solutions with function of $\eta$, i.e. $\theta_{1}(n)$ and $\theta_{2}(\eta)$, Eqs. (1) and (2) are reduced to the following ordinary differential equations:

$\theta_{1}^{\prime \prime}+2 \eta \theta_{1}^{\prime}=0$,

$\frac{a_{2}}{a_{1}} \theta_{2}^{\prime \prime}+2 \eta \theta_{2}^{\prime}=0$,

where ( $\boldsymbol{\prime}$ ) denotes the differentiation with respect to $n$. The boundary conditions (3), (4), and (5) and the initial condition (6) are reduced to the following three conditions:

$$
\begin{array}{ll}
\text { at } n=0 ; & \theta_{1}(\eta)=\theta_{f}, \\
\text { at } n=\frac{h(t)}{2 \sqrt{a_{1} t} ;} & \theta_{1}(\eta)=\theta_{2}(\eta)=\theta_{b},
\end{array}
$$


From Eq.(8), the solution which satisfies the boundary condition (10) is given as

$\theta_{1}(\eta)=k_{1} \int_{0}^{\eta} \exp \left(-x^{2}\right) d x+\theta_{f}$

and also, from Eq.(9), the solution which satisfies the boundary condition (12) is given as

$\theta_{2}(n)=k_{2} \sqrt{a_{2} / a_{1}} \int_{-\infty}^{\sqrt{a_{1} / a_{2}} \eta} \exp \left(-x^{2}\right) d x+\theta_{0}$

in which $k_{1}$ and $k_{2}$ are constants to be determined by the conditions at the boundary .

By substituting the boundary condition (11) into Eqs.(13) and (14), we obtain a relation

$k_{1} \int_{0}^{h(t) / 2 \sqrt{a_{1} t}} \exp \left(-x^{2}\right) d x+\theta_{f}=k_{2} \sqrt{a_{2} / a_{1}} \int_{-\infty}^{\sqrt{a_{1} / a_{2}} h(t) / 2 \sqrt{a_{1} t}} \exp \left(-x^{2}\right) d x+\theta_{0}=\theta_{b}$.

Since Equation (15) has to be satisfied for all values of the time, $h(t)$ must be proportional to $\sqrt{t}$. Therefore, the location of the boundary surface $h(t)$ is given as

$h(t)=2 \alpha \sqrt{a_{1} t}$,

where $\alpha$ is a constant.

From Eqs. (15) and (16), $k_{1}$ and $k_{2}$, are given as follows:

$k_{1}=\frac{\theta_{b}-\theta_{f}}{\int_{0}^{\alpha} \exp \left(-x^{2}\right) d x}$,

$k_{2}=\sqrt{a_{2} / a_{1}} \frac{\theta_{b}-\theta_{0}}{\int_{-\infty}^{\sqrt{a_{1} / a_{2}}} \alpha^{\alpha}\left(-x^{2}\right) d x}$.

Note that these equations involve an unknown constant $\alpha$ which should be determined by the matching condition at the boundary (7). By substituting Eqs.(13), (14), (16), (17), and (18) into Equation (7), we obtain a relation

$\frac{\exp \left(-\alpha^{2}\right)}{\int_{\alpha}^{0} \exp \left(-x^{2}\right) d x}-\frac{\lambda_{2}}{\lambda_{1}} \sqrt{a_{1} / a_{2}} \frac{\exp \left(-a_{1} \alpha^{2} / a_{2}\right)}{\int_{-\infty}^{\sqrt{a_{1} / a_{2}}} \begin{array}{l}\theta_{b}-\theta_{0} \\ \exp \left(-x^{2}\right) d x\end{array}}=\frac{2 \varepsilon \rho \alpha q}{\theta_{f}-\theta_{b}}=\frac{C_{p} \rho_{b}\left(\theta_{f}-\theta_{b}\right)}{,}$ 
where $\rho_{b}$ is the density of the upper region and $C_{p}$ is the specific heat of the upper region.

The values of $\alpha$ can be numerically obtained from Eq.(19). Therefore, the location of the boundary surface $z=h(t)$ is predicted by using Eq.(16). Figure 9 shows the variations of the boundary surface predicted using Eq.(16), with $\theta_{f}$ as a parameter. Thermal conductivities of the upper region and the lower region are given by a theoretical formulation [10] and the values of $\lambda_{1}$ and $\lambda_{2}$ used are $0.23 \mathrm{~W} / \mathrm{m} . K$ and $0.36 \mathrm{~W} / \mathrm{m}$. K. The
values of $a_{1}$ and $a_{2}$ used are $2.4 \times 10^{-7} \mathrm{~m}^{2} / \mathrm{s}$ and $2.3 \times 10^{-7} \mathrm{~m}^{2} / \mathrm{s}$, respectively. For comparison, the liquid level estimated using the methanol consumption in Fig.2 is also indicated by a solid line. Based on the results in Fig.9, the variations of methanol consumption are also evaluated and shown in Fig.10. For comparison, the experimental result is also indicated by a solid line.

As can be seen in Figs. 8 and 9 , the predicted results are qualitatively in good agreement with the experimental one. At an early stage of burning $(t=0-1200 \mathrm{~s})$ the temperature on the surface rises from 300 $\mathrm{K}$ to about $700 \mathrm{~K}$ (see Fig.4). Comparing the predicted value for $\theta_{\mathrm{f}}=500 \mathrm{~K}$ with the experimental one, they are fitted within $30 \%$ at $t=600 \mathrm{~s}$. $\mathrm{As}$ for the period of $t=1200 \sim 2400 \mathrm{~s}$, the temperature on the surface is about $740 \mathrm{~K}$. The difference between the predicted value for $\theta_{f}=740 \mathrm{~K}$ and the experimental one is only 20\%. Therefore, this simplified heat transfer model can well describe the experimental results not only qualitatively but also quantitatively. It seems that when the bead diameter is large, the consumption rate of the methanol in the bed is mainly controlled by the heat transfer in the porous bed.

\section{CONCLUSIONS}

The burning characteristics of methanol soaked in porous beds were experimentally investigated. The consumption rates of methanol and the the temperature distributions in the porous beds were measured. The following conclusions were obtained:

(1) Extinction occurred at 2500 4500 s after ignition. Only half of the initial methanol was consumed during combustion.

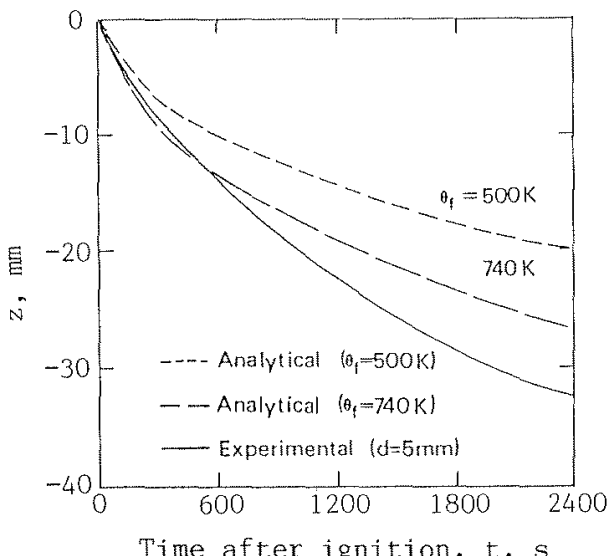

Time after ignition, $t, s$

FIGURE 9. Variation of the predicted boundary surface

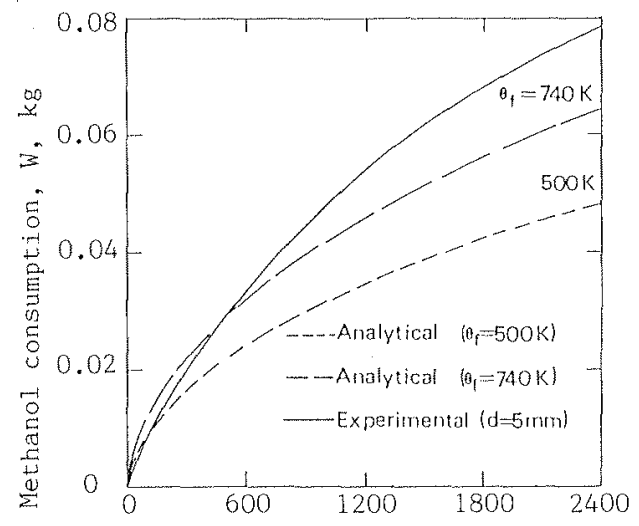

Time after ignition, $t, s$

FIGURE 10. Methanol consumption evaluated 
(2) The consumption rates of methanol were about $8 \times 10^{-5} \mathrm{~kg} / \mathrm{s}$ at ignition. They decreased with time, and they were about $1.5 \mathrm{~kg} / \mathrm{s}$ at extinction.

(3) The linear regression rates of the methanol were estimated to be $3 \times 10^{-5} \mathrm{~m} / \mathrm{s}$ and $0.6 \times 10^{-5} \mathrm{~m} / \mathrm{s}$ at ignition and extinction, respectively.

(4) Total weight of the methanol consumed and the consumption rate of the methanol seemed to increase with decreasing bead diameter.

(5) The temperature-time records indicated that the dry region expanded from the surface towards the bottom in the bed with time. The lower boundary of the dry region agreed with the liquid level evaluated in a simple way from the variations of weight for the large diameter beads, whereas they did not agree for the small diameter beads. This discrepancy in the bed of small beads seems to be due to the capillary force.

(6) A simplified model based on one-dimensional heat transfer was proposed to predict the consumption rate of methanol for large beads. The predicted results were in qualitative agreement with the experimental results. Therefore, the fuel consumption rate on the large beads bed was found to depend mainly on the heat transfer in the bed.

\section{REFERENCES}

1. Takeno,K., and Hirano,T.," Flame Spread over Porous Solid Soaked with a Combustible Liquid", Twenty-first Symposium (International) on Combustion, The Combustion Institute, pp. 75-81, 1986 .

2. Ishida,H., "Flame Spread over Ground Soaked with Highly Volatile Liquid Fuel", Fire Safety Journa1, 13, 115-123, 1988 .

3. Takeno,K., and Hirano,T., "Behavior of Combustible Liquid Soaked in Porous Beds During Flame Spread", Twenty-second Symposium (Internationa1) on Combustion, The Combustion Institute, pp.12231230,1988 .

4. Suzuki,T., and Hirano,T., "Flame Spread over Fuel Soaked Sand in an Opposed Air Stream", Fire Safety Science, Proceedings of the Second Symposium (International) on Fire Safety Science, Hemisphere, pp.199208,1989 .

5. Blinov,V.I., and Khudiakov,G.N., "Certain Laws Governing Diffusive Burning Liquids", Academiia Nauk, SSSR Doklady, 5, 1094-1098, 1957.

6. Hottel,H.C., "Certain Laws Governing Diffusive Burning Liquids", Fire Research Abstracts and Reviews, 1, 41-44, 1959.

7. Burges,D.S., Strasser,A., and Grumer,J., "Diffusive Burning of Liquid Fuels in Open Trays", Fire Research Abstracts and Reviews, 3, $177-192$, 1961

8. Akita,K., and Yumoto, T., "Heat Transfer in Small Pools and Rates of Burning of Liquid Methano1", Tenth Symposium (Internationa1) on Combustion, The Combustion Institute, pp. 943-950, 1965 .

9. De Ris,J., and Orloff,L., "A Dimensionless Correlation of Pool Burning Data", Combustion and Flame, 18, 381-388, 1972.

10. Kunii, D. and Smith, J,M ., "Heat transfer Characteristics of Porous Rocks", American 'Institute of Chemical Engineering Journal, $6,71-77,19 \overline{60}$. 